\title{
Interactive, hands-on training for hospital workers increases level of special pathogen preparedness
}

\author{
Authors: \\ Eleanor Tolf \\ Intern, System-wide Special Pathogens Program, NYC Health + Hospitals \\ Georgetown University, B.S. \\ Syra Madad, DHSc, MSc and MCP
}

Senior Director, System-wide Special Pathogens Program, NYC Health + Hospitals

\author{
For correspondence: \\ Syra Madad \\ NYC Health + Hospitals \\ 125 Worth Street, Suite 412 \\ New York, NY 10013 \\ syramadad@gmail.com
}

Short running title: Interactive training increases SP preparedness.

\begin{abstract}
Objective: The purpose of this evaluation is to determine the effect of intensive, interactive training on hospital workers' preparedness for special pathogen cases by utilizing the Frontline Facility Special Pathogens Training Course created by New York City Health + Hospitals (NYC H+H).

Methods: An 8-hour, bi-monthly course conducted between 2018 and 2019 was offered to health care employees throughout the United States Department of Health and Human Services Region 2, mostly from NYC H+H. Evaluation included multiple choice pre and post exams, a 26-question survey about level of preparedness before and after the training, and follow up interviews focused on changes in facility protocols.

Results: $61 \%$ of survey respondents indicated that they had never attended a hospital-sponsored special pathogens training before. After the training, there was a 53.3\% report rate of feeling "very prepared," compared to $14.6 \%$ before the training. Additionally, there was an $11 \%$ improvement in test scores. $77 \%$ of respondents also reported that their facility had changed protocols or processes relating to topics covered in the course after their training date.

Conclusions: Survey participants reported general satisfaction with the course as well as an increased level of preparedness for special pathogen cases. Together, the results of the exams, survey, and interviews suggest that this interactive, mixed-method training increases special pathogen preparedness across different healthcare sectors.
\end{abstract}

Keywords: preparedness; special pathogens; Ebola; PPE; training

\section{Introduction}

In a highly globalized world, the threat of special pathogen outbreaks is omnipresent. In the year 2018, there were at least seven reported special pathogens outbreaks around the world to the World Health Organization (WHO, 2018). Special pathogens are those that are associated with high morbidity and/or mortality, have a high likelihood of secondary cases, lack an effective vaccine, treatment, prophylaxis or treatment and/or might prompt the use of a biocontainment unit (Madad et al, 2019). They include viral hemorrhagic fevers, such as Ebola and Lassa Fever, as well as severe respiratory special pathogens, such as Middle East Respiratory Syndrome and Severe Acute Respiratory Syndrome. Special pathogens pose a major threat to the general public and in healthcare settings where specific and meticulous processes must be rapidly implemented to ensure early identification, isolation, effective infection control practices and notification to public health authorities of infected patients to prevent disease transmission. 
Several factors can contribute to the emergence or re-emergence of special pathogen diseases such as population growth and human behavior, international travel, climate and ecological change, microbial adaptation, economic development, and breakdown in public health measures. Combinations of these factors may lead to fast and widespread transmission to populations across the world, resulting in potentially large-scale epidemics or pandemics.

In March of 2014, the WHO reported Ebola cases in rural Guinea. The next 2 years represented the largest Ebola epidemic in history, including numerous cases in Liberia, Sierra Leone, Mali, Nigeria, Senegal, Spain, the United Kingdom, and the United States. During the outbreak, many public health experts and academics expressed concerns about the United States' ability to properly manage patients with suspected and confirmed Ebola or other special pathogens (Aronczyk, 2018). These concerns became a reality in September 2014. Thomas Eric Duncan, a Liberian national visiting the United States, was admitted to Texas Heath Presbyterian Hospital in Dallas, Texas where he was diagnosed with Ebola Virus Disease (EVD). During his time as an inpatient, two nurses were exposed to the virus and both were diagnosed with EVD in October 2014. Within the same month, Dr. Craig Spencer was diagnosed with EVD in New York and treated in NYC H+H's Bellevue Hospital. Furthermore, during the 2014 outbreak in the United States, there were only four high-level isolation units (HLIU) in the country (Qureshi, 2016). In April of 2015, the CDC contacted 55 Ebola Treatment Centers (ETCs) in the U.S. and determined that only 3 had adequate preexisting biocontainment units and the other 52 needed to undertake extensive changes, including "development of plans, recruitment of facility leadership, recruitment and training of a multidisciplinary team of volunteers, and purchase of specialized supplies and equipment" (Herstein, 2016). These developments cost the ETCs nearly $\$ 54$ million. The outbreak exposed the numerous gaps that exist in the country's preparedness plans.

As a hub for both national and international travel, New York City plays a vital role in protecting the United States against transmission of special pathogens. NYC H+H is the largest public health care system in the United States and one of the few healthcare systems in the country capable of treating an Ebola patient (About NYC Health + Hospitals, 2018). With 60 patient care sites across the five boroughs of New York City including one Ebola and Other Special Pathogens Treatment Center, NYC H+H is an important first line of defense for any outbreak in the city; however, from a sample of $73 \mathrm{NYC} \mathrm{H}+\mathrm{H}$ administrators, physicians, and nurses, $85.4 \%$ rated their facility as somewhat prepared or unprepared to combat an outbreak of a special pathogen.

Primary healthcare providers from NYC $\mathrm{H}+\mathrm{H}$ have expressed concern with the seemingly too narrowed focus on identification. When it comes to the goal in special pathogen preparation to "Identify, Isolate, Inform," commented one physician, "We're good at identifying, but if we are strong in just one area, we will fail." Staff rarely work in the level of personal protective equipment (PPE) that would be required for special pathogens. An outbreak will also place staff in an environment of much higher stress and less preparation than they are accustomed. Training in these unusual conditions is necessary to prepare for the real-life conditions of a special pathogen case. The Frontline Facility Special Pathogens Training Course, created by the hospital systems' Special Pathogens Program, is an 8-hour course offered to health care workers throughout the NYC H+H healthcare delivery system and The United States Health and Human Services Region 2 partners, which includes New York, New Jersey, Puerto Rico, and the Virgin Islands (Region 2 HHS, 2018) and is open to physicians, nurses, ancillary/support staff. The course mixes presentations, simulations, and discussions in an attempt to better prepare healthcare employees for an unexpected special pathogen case. The goal is to equip NYC $\mathrm{H}+\mathrm{H}$ administrators and healthcare providers with centralized skills in order to effectively identify, isolate, rapidly implement effective infection control measures and inform appropriate stakeholders in the event of a special pathogen case(s).

\section{Methods}

The 8-hour course for frontline facility's, offered bimonthly; includes three modules, and culminates in group analysis of the participants' performance and discussion of special considerations, such as pediatric, geriatric, and behavioral health. It does so through presenting decision support tools and resources, discussing best practices and basic infection control strategies, and providing immersive live scenarios while participants are dressed in high level PPE. The course covers the practice of donning/doffing PPE and participating in clinical stations including MERS workup on a mid-fidelity mannequin, immediate spill remediation of a bodily fluid spill, removal of contaminated PPE and how to properly transfer and transport patients while wearing the high-level PPE attire. Module 1 covers "Identify, Isolate, Inform" and basic infection control practices, module 2 covers working in Special Pathogens Level 1 PPE with live patient care simulations, and module 3 covers working in Special Pathogens Level 2 VHF PPE with live patient care simulations. 
The following cohort analysis involves a survey, pre and post-course examinations, and interviews of the course participants [Sup. Fig 1]. The survey, given no less than 2 weeks after completion of the course, evaluates participants' prior preparation for and awareness of special pathogen cases, the course's ability to meet expectations, and how the course changed their knowledge and/practices.

In addition to a written, survey-based approach, in-depth interviews were conducted with 10 participants 1-2 weeks after completion of the survey, centered around the following topics: personal feelings of preparedness, evaluations of others and team dynamics, facility protocols, and facility preparedness/equipment [Supp. Fig 2].

Finally, quizzes covering knowledge of special pathogen preparedness were given on the day of training immediately before and after the course. They were evaluated for quantitative improvement in knowledge.

The end result of these surveys and interviews is a mixed-methods report, combining both quantitative analysis of the participants' changes in knowledge and their reported (survey-based) preparedness levels, as well as qualitative discussion based on interviews.

\section{Results}

\section{Before the Training}

According to data collected in the survey [Supp. Fig 3], the majority of the 50 respondents work in a hospital, while the others either work in healthcare management, such as a government agency, or in a non-hospital healthcare facility, such as a nursing home. Their employment includes healthcare practitioners, public servants, and other non-traditional healthcare vocations, including educators, janitorial workers, and hospital administrators. Healthcare practitioners, including nurses and physicians, made up nearly half of the respondents.

The majority of respondents, $66.6 \%$, indicated that their facility tests for competency in special pathogen preparedness every year or 6 months, while only 5 reported that their facility did not test for competency. Most, 83.7\%, also indicated they "often" stay updated with news about special pathogens [Table 1]. Additionally, 61\% of the 41 participants reported that they had never attended a hospital-sponsored special pathogens training course. Only half of the remaining 39\% reported attending a course not presented by the NYC H+H Special Pathogens Team [Fig. 1].

Table 1. How often does your facility test for competency in special pathogens preparedness? $\mathrm{n}=48$.

\begin{tabular}{|c|c|c|c|c|c|}
\hline Every year & $\begin{array}{c}\text { Every } 6 / \text { few } \\
\text { months }\end{array}$ & \multicolumn{2}{|c|}{ Every month } & I don't know & $\begin{array}{l}\text { My facility doesn't } \\
\text { test for competency }\end{array}$ \\
\hline $20(\mathbf{0 . 4 2})$ & $12(\mathbf{0 . 2 5})$ & \multicolumn{2}{|c|}{$1(\mathbf{0 . 0 2})$} & $9(\mathbf{0 . 1 9})$ & $6(\mathbf{0 . 1 2})$ \\
\hline \multicolumn{6}{|c|}{ How often are you updated with news and information about special pathogens? $n=48$} \\
\hline Very often & \multicolumn{2}{|c|}{ Somewhat often } & \multicolumn{2}{|c|}{ Not very often } & Never \\
\hline $23(\mathbf{0 . 4 8})$ & \multicolumn{2}{|c|}{$17(\mathbf{0 . 3 5})$} & \multicolumn{2}{|c|}{$8(\mathbf{0 . 1 7})$} & $0(\mathbf{0 . 0 0})$ \\
\hline \multicolumn{6}{|c|}{ Had you already attended a hospital-sponsored special pathogens training course? $\mathrm{n}=48$} \\
\hline \multirow{2}{*}{\multicolumn{2}{|c|}{$\frac{\text { No }}{29(\mathbf{0 . 6 1})}$}} & \multicolumn{3}{|c|}{ Yes (this one) } & es (another one) \\
\hline & & \multicolumn{2}{|c|}{$11(\mathbf{0 . 2 3})$} & & $8(\mathbf{0 . 1 6})$ \\
\hline
\end{tabular}

Table 1. The majority of respondents indicated that their facility tested for special pathogen preparedness competency every year or every 6 months and kept updated with relevant news and information somewhat to very often. The majority of participants had never attended a hospital-sponsored special pathogens training course; of those who did, 11 of 19 had previously attended the Frontline Facilities Special Pathogens Training Course.

Since much of the training course involves learning to work in PPE specific to special pathogens, the survey asked about the participant's' prior experience. 33\% reported that working in such extensive PPE was somewhat new and $24 \%$ reported that it was very new [Fig 2]. In addition to PPE, patient transfer was the topic that most respondents reported being least comfortable with [Fig 1]. Patient transfer is the act of physically lifting, packaging and moving a suspected or confirmed patient with a special pathogen disease from a hospital bed to a transport vehicle such as a stretcher or wheelchair while minimizing contamination risk and avoiding risks or injuries to the patient or staff. 


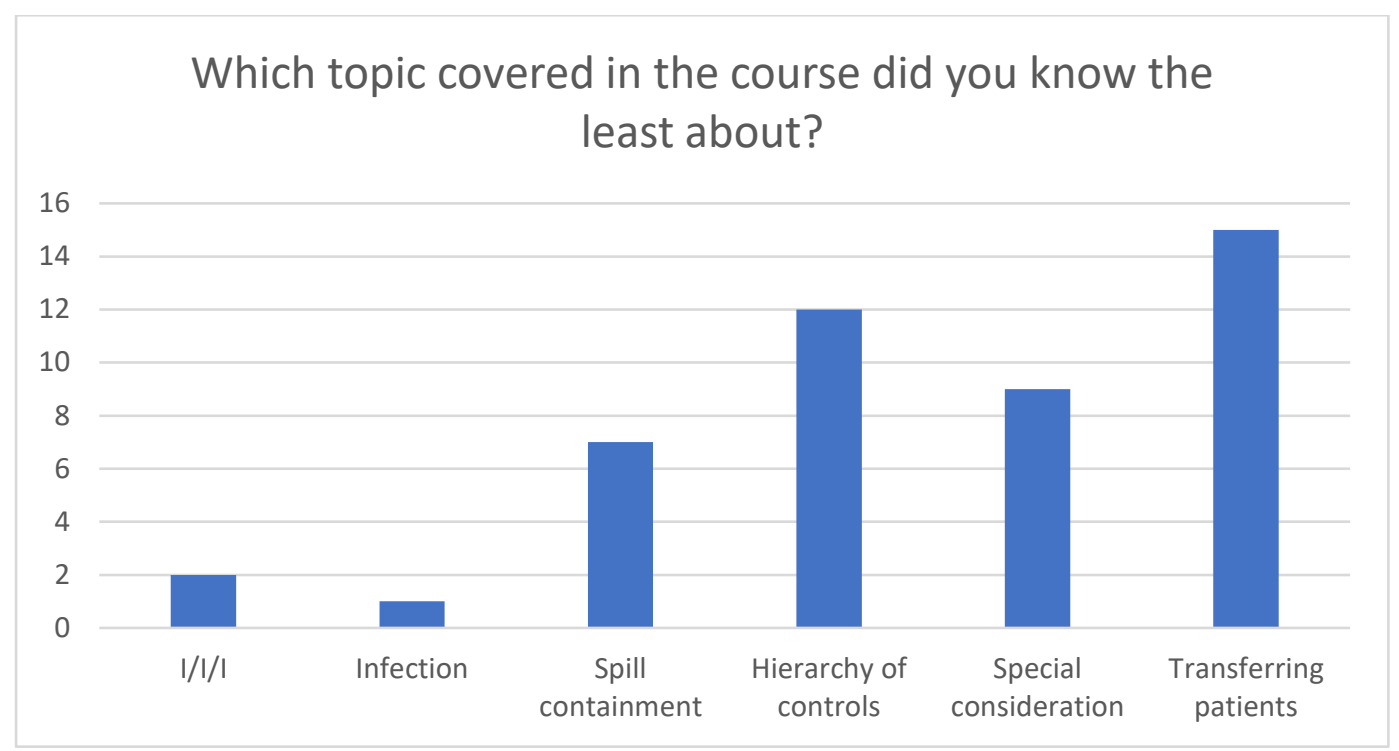

Figure 1. Of the 46 participants who answered this question, the topics most indicated as being new to participants were hierarchy of control (12), special consideration (9), and patient transfer (15). I/I/I represents the topic of "Identify, Isolate, Inform.".

\section{Was working in such extensive PPE new for you? \\ - Very new - Somewhat new Not new at all}

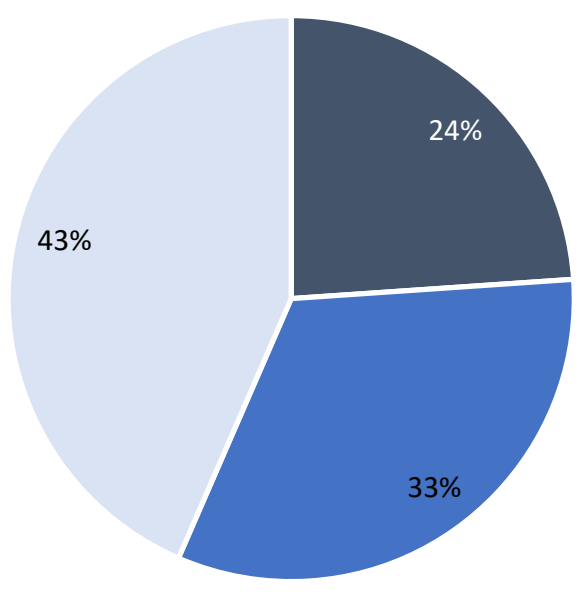

Figure 2. Of the 46 participants who answered this question, 57\% indicated that working in SP Level 1 and Level 2 was new, with $24 \%$ labeling it as very new. .

Change in Preparedness Level

Self-reported levels of preparedness shifted, with 53.3\% respondents feeling "very prepared" after the training, compared to the initial $14.6 \%$ of the same response [Fig 3]. Almost the entirety of the rest of the responses after the training were of feeling "somewhat prepared," with only one participant selecting "somewhat unprepared." Before the training, most respondents reported feeling either "somewhat prepared" or "somewhat unprepared," with 5 reporting feeling "very unprepared." In addition to personal feelings of preparedness, most participants (77\%) reported that their facility has changed their protocols surrounding special pathogen preparedness after the course [Fig 4]. Most reported protocol changes involved special consideration, such as geriatric or prenatal issues, or Special Pathogen PPE Level 1 and 2 [Table 1]. These changes were followed up in-depth during the interviews. 


\section{How prepared do you feel to face a special pathogens case in your facility?}

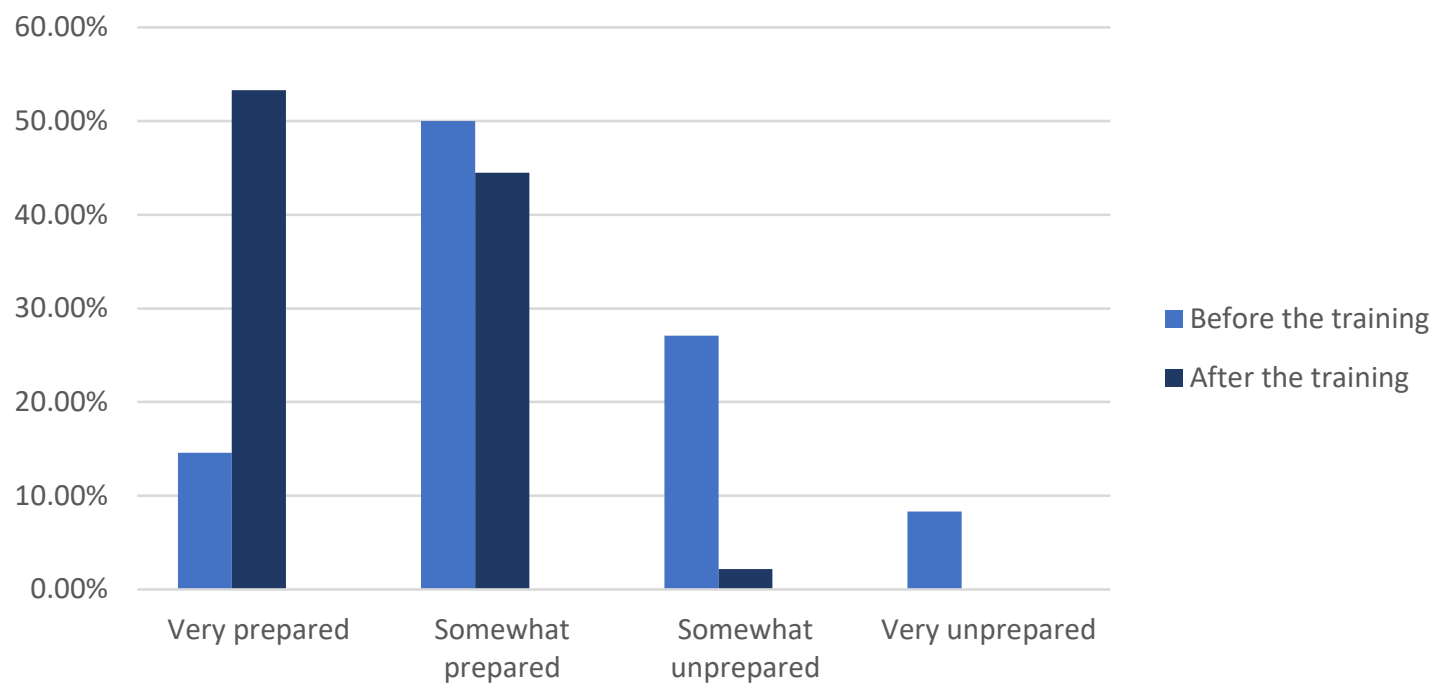

Figure 3. Of the 45-48 participants, most reported feeling somewhat prepared or somewhat unprepared before the training, with some feeling very prepared or unprepared. After the training, almost every participant reported feeling prepared, with the majority feeling very prepared.

\section{After the training, has your facility changed protocols surrounding any of the topics covered in the training?}

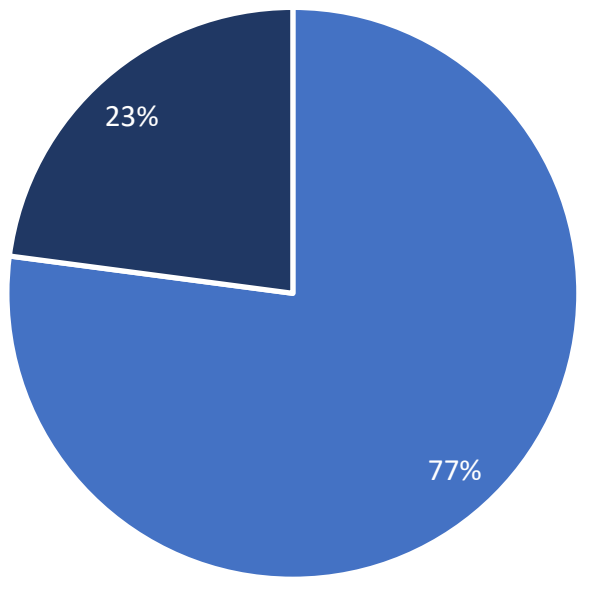

Figure 4. Of the 48 respondents, 77\% reported that their facility had changed their special pathogen protocols after they had taken the training.

Preliminary data from pre and post exams show an overall increase in knowledge, with an average improvement of $20 \%$ between tests [Fig 5]. The improvement varied greatly between the first 20 classes, with class 14 showing a $26.5 \%$ improvement and class three showing a $1.30 \%$ improvement. 


\begin{tabular}{|c|c|c|c|c|c|}
\hline \multirow[b]{2}{*}{ Class Number } & \multicolumn{2}{|c|}{ Pre Test Scores } & \multicolumn{2}{|c|}{ Post Test Scores } & \multirow{2}{*}{$\begin{array}{c}\text { Improvement } \\
\text { Between Each Test }\end{array}$} \\
\hline & Participant \# & Total Average & Participant \# & Total Average & \\
\hline Class One & 7 & $81.4 \%$ & 7 & $88.6 \%$ & $7.2 \%$ \\
\hline Class Two & 4 & $70.0 \%$ & 4 & $90.0 \%$ & $20.0 \%$ \\
\hline Class Three & 9 & $76.7 \%$ & 10 & $78.0 \%$ & $1.3 \%$ \\
\hline Class Four & 11 & $69.0 \%$ & 11 & $85.0 \%$ & $16.0 \%$ \\
\hline Class Five & 13 & $67.7 \%$ & 18 & $80.6 \%$ & $12.9 \%$ \\
\hline Class Six & 10 & $76.0 \%$ & 11 & $81.0 \%$ & $5.0 \%$ \\
\hline Class Seven & 10 & $80.0 \%$ & 13 & $83.0 \%$ & $3.0 \%$ \\
\hline Class Eight & 10 & $77.0 \%$ & 11 & $87.3 \%$ & $10.3 \%$ \\
\hline Class Nine & 12 & $77.0 \%$ & 12 & $87.3 \%$ & $10.3 \%$ \\
\hline Class Eleven & 12 & $78.4 \%$ & 12 & $91.0 \%$ & $12.6 \%$ \\
\hline Class Ten & 12 & $79.2 \%$ & 12 & $82.0 \%$ & $2.8 \%$ \\
\hline Class Twelve & 9 & $77.8 \%$ & 9 & $90.0 \%$ & $12.2 \%$ \\
\hline Class Thirteen & 6 & $76.7 \%$ & 6 & $68.0 \%$ & $-8.7 \%$ \\
\hline Class Fourteen & 13 & $61.5 \%$ & 13 & $88.0 \%$ & $26.5 \%$ \\
\hline Class Fifteen & 16 & $71.9 \%$ & 14 & $88.0 \%$ & $16.1 \%$ \\
\hline Class Sixteen & 14 & $70.7 \%$ & 16 & $93.0 \%$ & $22.3 \%$ \\
\hline Class Seventeen & 4 & $62.5 \%$ & 5 & $88.0 \%$ & $25.5 \%$ \\
\hline Class Eighteen & 6 & $78.0 \%$ & 6 & $90.0 \%$ & $12.0 \%$ \\
\hline Class Nineteen & 7 & $77.1 \%$ & 6 & $93.0 \%$ & $15.9 \%$ \\
\hline Class Twenty & 6 & $71.1 \%$ & 4 & $88.0 \%$ & $16.9 \%$ \\
\hline Total & 191 & $74.0 \%$ & 200 & $86 \%$ & $12 \%$ \\
\hline
\end{tabular}

Figure 5. Test results from the first twenty courses, showing percentage correct immediately before and immediately after the 20 questions exams. The questions were different, but they covered the same topics. The average improvement between tests for all classes was $20 \%$.

Satisfaction

The majority of respondents, $80.4 \%$, agreed that the course covered the expected topics [Table 2]. 95.7\% indicated they believed their colleagues would benefit from taking the same course, with the other respondents selecting "probably" or "maybe." Finally, 78.2\% reported that they would be interested in taking yearly refresher course; $19.6 \%$ reported maybe and $2.2 \%$ reported no [Table 2$]$.

\begin{tabular}{|c|c|c|c|}
\hline \multicolumn{2}{|c|}{ Did the course go over all the topics you expected/hoped? $\mathrm{n}=46$} \\
\hline Yes & No & \multicolumn{2}{c|}{ Had no expectations } \\
\hline $37(\mathbf{0 . 8 1})$ & $1(\mathbf{0 . 0 2})$ & \multicolumn{1}{c|}{$\mathbf{( 0 . 1 7 )}$} \\
\hline \multicolumn{2}{|c|}{ Do you think colleagues would benefit from taking this course? $\mathrm{n}=46$} \\
\hline Very much & Probably & Maybe & No, it's unnecessary \\
\hline $34(\mathbf{0 . 7 4})$ & $10(\mathbf{0 . 2 2})$ & $2(\mathbf{0 . 0 4})$ & 0 \\
\hline Would you be interested in taking a shorter, refresher course every year? $\mathrm{n}=42$ \\
\hline Yes & Maybe & No \\
\hline $36(\mathbf{0 . 7 8})$ & $9(\mathbf{0 . 2 0})$ & $1(\mathbf{0 . 0 2})$ \\
\hline
\end{tabular}

Table 2. 42 participants answered questions about overall satisfaction with the training course. The majority reported that the course covered what they expected (81.0\%), that their colleagues could benefit from it (73.8\%), and that they would be interested in taking a yearly refresher course $(76.2 \%)$.

\section{Discussion}

After 20 course offerings, survey participants reported not only general satisfaction with the course, but also an increased level of preparation for a special pathogen case. Specifically, greatest improvements in preparation were made in the areas of special considerations and special pathogen-specific PPE. Interview responses elaborated on the course's 
ability to increase participants' confidence and the course's ability to affect change in facility protocol. Together, these results suggest that this interactive, mixed-method training increases special pathogen preparedness across different healthcare sectors.

\section{Before the training}

The demographic data collected from the survey indicates that many of those that self-select to take the course are hospital workers, but not necessarily nurses and physicians. Follow-up interviews with administrators and managers revealed that many of these professionals felt excluded from normal hospital preparedness trainings and were worried about their role in the event of a special pathogens outbreak. Environmental service employees found it especially important to take this course as they will also be expected to work in the relevant PPE.

Questions about prior preparedness interventions indicate that both hospital workers and the hospital themselves are already taking active steps to be prepared. Most facilities are testing regularly for PPE competency and the employees themselves self-report staying up to date with relevant news and information; however, most reported never attending a hospital-sponsored special pathogens training course before, indicating that facilities may be testing for preparedness without offering the necessary education beforehand.

Respondent's report of inexperience working in PPE and patient transfer reinforced the need for this course, as the second and third module focus heavily on moving the patient and performing procedures in the high level special pathogens PPE.

\section{Change}

Self-assessments of preparedness before and after the training suggest a dramatic shift from the majority of participants feeling somewhat prepared and somewhat unprepared before the training, to almost the entirety of the respondents indicating feelings of preparedness, with the majority feeling "very prepared." Although this result is self-reported, it is incredibly promising as it indicates that the workers left the training feeling more confident in their abilities, which will help them work better as a team member in the case of a special pathogens case.

Results from the pre- and post-examinations also indicate that there is objective improvement in knowledge surrounding special pathogen preparedness. Some of the most unexpected but most promising results from the survey were the questions involving changes in facility protocol. The majority of respondents indicated that their facility had changed their facility protocols related to special pathogens, most often those involving special considerations for pediatric, geriatric and behavioral health patients and special pathogen specific PPE after they had taken this training.

Follow-up interviews with physicians and administrators from different healthcare facilities in Region 2 were conducted to more deeply evaluate how hospitals instated changes in protocol after their employees attended the class. Most hospital staff reported that the largest protocol change was in PPE training and equipment availability. Although all interviews reported having prior training with PPE, all reported that these preparations consisted of online training courses and demonstrations; one described the state of PPE protocol as "woe-fully underdeveloped." Reported changes after the Frontline course included more accessibility to the equipment detailed in the training course and instituting active donning and doffing training drills, rather than relying on online modules.

\section{Satisfaction}

The majority of respondents indicated that the course was what they expected, that it would benefit their colleagues, and that they would be interested in taking a yearly refresher course. Follow-up interviews reported no dissatisfaction with the course. When prompted for recommendations for alternations, only one interviewee requested including "applicable honesty," a discussion of what is realistic for clinicians and making sure not to undermine the existing knowledge and experience they already have about the subject.

\section{Conclusions}

The growing threat of special pathogens poses a significant challenge to healthcare systems. As incidences of special pathogen diseases continue to increase around the world, it is increasingly important for all healthcare workers to prepare for the nearly inevitable next outbreak. This includes strategies and decision support tools in aiding for rapidly identifying, isolating, implementing effective infection control practices and applying appropriate PPE and notifying relevant stakeholders for patients with suspected or confirmed special pathogens disease. The Frontline Facility Special 
Pathogens Training Course addresses all these topics and has provided the opportunity for hospital staff to engage with the pressing reality of a special pathogens case(s).

\section{References}

World Health Organization. (2018). Disease Outbreak News. https://www.who.int/csr/don/archive/year/2018/en/

Aronczyk, A. (2018). Germ city: How SARS changed the face of air travel | WNYC | new york public radio, podcasts, live streaming radio, news. Retrieved from www.wnyc.org/story/germ-city-how-sars-changedface-air-travel/.

“Ebola Virus Disease in the United States ." Ebola Virus Disease: From Origin to Outbreak, by Adnan Qureshi, Elsevier Science, 2016.

Herstein, J. J., Biddinger, P. D., Kraft, C. S., Saiman, L., Gibbs, S. G., Smith, P. W...Lowe, J. J. (2016). Initial Costs of Ebola Treatment Centers in the United States. Emerging Infectious Diseases, 22(2), 350-352. https://dx.doi.org/10.3201/eid2202.151431.

About NYC health + hospitals. (2018). Retrieved from https://www.nychealthandhospitals.org/about-nychealth-hospitals/

Region 2 HHS. (2018). Retrieved from https://www.hhs.gov/about/agencies/iea/regional-offices/region-2/index.html 